\title{
La Grama Bermuda para Patios de Florida ${ }^{1}$
}

\author{
Laurie E. Trenholm, Marco Schiavon, J. Bryan Unruh, and Travis W. Shaddox ${ }^{2}$
}

La grama o césped Bermuda (Cynodon spp.) está entre las especies macrotermas más utilizadas. Las especies mejoradas y de textura fina de Bermuda son utilizadas en todo el Sur de Estados Unidos, en campos de golf, canchas deportivas y en áreas paisajísticas residenciales y comerciales donde se requiere de una cobertura del suelo densa y de textura fina. Generalmente, estas especies son híbridos de grama Bermuda común (Cynodon dactylon) y grama Bermuda africana (Cynodon transvaalensis) (Figura 1). Sin embargo, están disponibles cultivares mejorados de Bermuda común que pueden producir un césped de buena calidad en falta de sombra. Estas variedades tienen hojas más gruesas y normalmente no poseen la calidad visual de las variedades de textura fina, pero tampoco requieren un mantenimiento tan alto. Esta publicación está destinada a propietarios de casas y encargados de pasto y césped que manejan grama Bermuda en el paisaje de Florida.

\section{Ventajas}

La grama Bermuda produce un césped vigoroso, verde medio oscuro y denso, que está bien adaptado a la mayoría de los suelos y climas de Florida. La grama Bermuda posee excelente tolerancia al pisoteo, a la sequía, y a la salinidad, y también puede tolerar temperaturas bajas entrando en estado de hibernación. Se establece muy rápidamente superando la mayoría de las malezas. Se puede propagar utilizando piezas cuadradas o rectangulares (sod), ramitas (sprigs), o también se pueden conseguir semillas de algunas variedades mejoradas. La grama Bermuda es una de las mejores opciones en situaciones donde ninguna forma de riego (automática o manual) esté disponible.

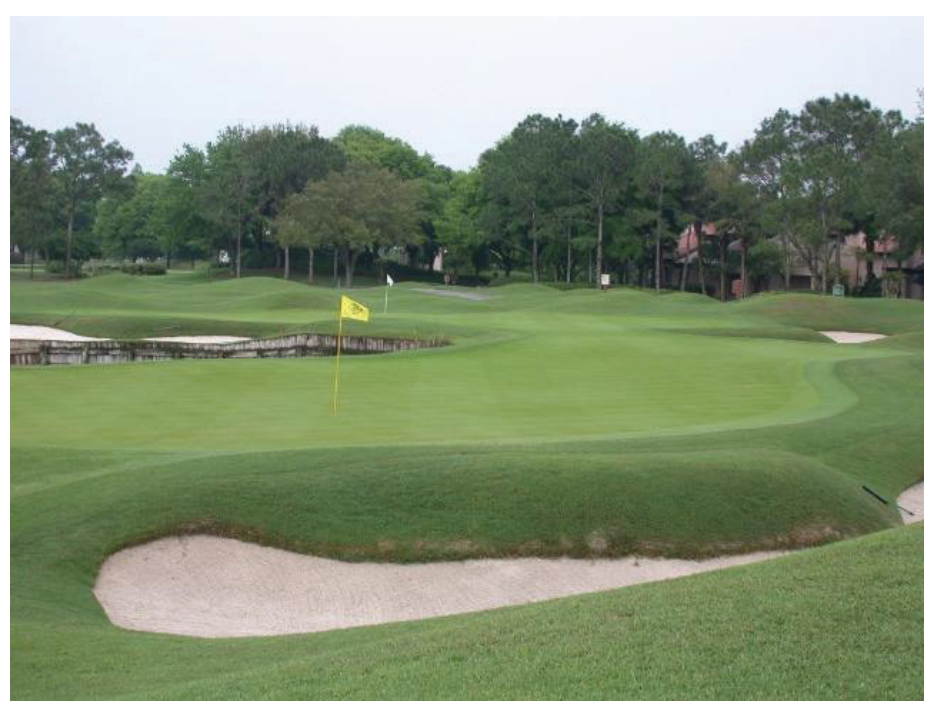

Figura 1. Grama Bermuda hibrida en un campo de golf.

Credits: J. Bryan Unruh, UF/IFAS

\section{Desventajas}

Los cultivares comúnmente usados en los campos de golf y las canchas deportivas requieren niveles de mantenimiento más altos comparados con otras especies utilizadas para patios. Además, poseen baja tolerancia a varios insectos, enfermedades y nemátodos que pueden limitar sus usos. Esta especie crece muy agresivamente gracias a sus estolones (ramitas arriba del suelo), y rizomas (ramitas

1. This document is ENH19s, one of a series of the Environmental Horticulture Department, UF/IFAS Extension. Original publication date July 2021. Visit the EDIS website at https://edis.ifas.ufl.edu for the currently supported version of this publication.

2. Laurie E. Trenholm, professor (retired), Environmental Horticulture Department, UF/IFAS Extension, Gainesville, FL; Marco Schiavon, assistant professor, turf and sod, Environmental Horticulture Department, UF/IFAS Ft. Lauderdale Research and Education Center, Davie, FL; J. Bryan Unruh, professor and associate center director, Environmental Horticulture Department, UF/IFAS West Florida REC, Jay, FL; and Travis W. Shaddox, former assistant professor of turfgrass, Environmental Horticulture Department, UF/IFAS Ft. Lauderdale REC, Davie, FL; UF/IFAS Extension, Gainesville, FL 32611.

The Institute of Food and Agricultural Sciences (IFAS) is an Equal Opportunity Institution authorized to provide research, educational information and other services

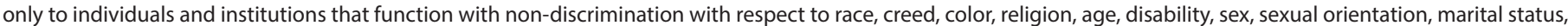
national origin, political opinions or affiliations. For more information on obtaining other UF/IFAS Extension publications, contact your county's UF/IFAS Extension office. U.S. Department of Agriculture, UF/IFAS Extension Service, University of Florida, IFAS, Florida A \& M University Cooperative Extension Program, and Boards of County Commissioners Cooperating. Nick T. Place, dean for UF/IFAS Extension. 
abajo del suelo) y puede invadir canteros y huertas muy rápidamente. El crecimiento muy agresivo puede causar acolchonamiento (thatch). La grama Bermuda normalmente no mantiene su color verde en baja temperaturas y posee escasa tolerancia a la sombra.

\section{Cultivares propagados}

\section{vegetativamente (por piezas y ramitas)}

\section{'Tifway', ‘TifGrand', y 'TifSport'}

Estos híbridos son utilizados en campos de golf y canchas deportivas. Estos cultivares requieren niveles de mantenimiento muy altos (por ejemplo, más fertilización y químicos, frecuencia de corte muy alta, etc.) y tendrían que estar manejados por empresas de mantenimiento de césped profesionales. 'TifGrand' requiere menos horas de sol directo y es menos sensible a los daños causados por grillos-topo (Scapteriscus spp.) comparado con 'Tifway' y 'TifSport'.

\section{'Latitude 36'}

'Latitude 36' es un híbrido relativamente nuevo desarrollado en Oklahoma State University. Se destaca por su habilidad a sobrevivir en climas fríos comparados con otros cultivares. Es más resistente al patógeno Ophiosphaerella, agente causal de la enfermedad Spring Dead Spot. 'Latitude 36' está comercializado en Florida y presenta buena calidad, sin embargo, es propenso a estar dañado por nemátodos. No es tan agresivo como otros cultivares.

\section{'TifTuf'}

Este es un nuevo híbrido proveniente de la Universidad de Georgia. Este cultivar es muy tolerante a la sequía, pero requiere niveles de mantenimientos similares a los de 'Tifway', 'TifGrand' y 'TifSport.' 'TifTuf' retiene su color en el otoño por más tiempo, y su respuesta a la sombra es comparable a la de 'TifGrand'.

\section{'Celebration'}

'Celebration' es una variedad versátil utilizada en campos de golf, canchas deportivas y patios. Tiene hojas de textura fina y buena calidad, pero no requiere tanto mantenimiento como los otros cultivares de textura fina. Posee buena tolerancia al tráfico y tolera un poco más de sombra que los otros cultivares. 'Celebration' se puede cortar a 1-2 pulgadas cuando se utiliza en patios con cortadora de césped rotativa. Cortar a alturas superiores a 2 pulgadas podría resultar en apariencia irregular.

\section{'Bimini'}

'Bimini' es un cultivar muy nuevo en el mercado de Florida. Su potencial de crecimiento se encuentra entre medio de 'Celebration' y los otros híbridos. Fue muy bien recibido por la industria del golf, y hay evidencia prematura de que puede usarse para distintas aplicaciones: golf, canchas deportivas y también patios. La altura de corte sería similar a la de 'Celebration'.

\section{'NorthBridge'}

Al igual que 'Latitude 36, 'NorthBridge' fue desarrollada en Oklahoma State University. Esta variedad es tolerante al frío y reverdece (green-up) pronto en la primavera, saliendo de su estado de hibernación muy pronto al principio del año.

\section{'PremierPRO'}

'PremierPRO' es un cultivar híbrido de textura fina desarrollado en Texas A\&M University. De momento se puede encontrar solo en el sur de Florida; este cultivar se puede utilizar en campos de golf, campos deportivos, y patios. La altura de corte recomendada varía entre 0.5 y 2.5 pulgadas. 'PremierPRO' posee crecimiento lateral agresivo que lo ayuda con el establecimiento y la recuperación después de estar dañado.

\section{Cultivares sembrados}

Las tiendas y comercios normalmente venden semillas de Bermuda común (Figura 2). Dos de las variedades propagadas por semillas de buena calidad por el mercado de Florida son 'Princess 77' y 'Sultan'. A menudo se encuentran mezclas de dos o tres variedades que incluyen cultivares tipo 'Arizona Common', 'Riviera,' 'Cheyenne, 'Sahara,' 'Sundevil', 'Jackpot' y otros. Las variedades contenidas en esas mezclas pueden cambiar año a año dependiendo de la disponibilidad de las semillas. Estas variedades mejoradas poseen un color verde oscuro, raíces profundas, más densidad foliar y una textura foliar menos gruesa que las viejas selecciones de Bermuda común (como por ejemplo 'Arizona Common'). A pesar de que estas variedades son adecuadas para patios, deportes y bordes de las rutas, su calidad es generalmente peor que la de los cultivares propagados vegetativamente. Cabe mencionar que el desempeño de las mezclas de cultivares aún no ha sido evaluado por la Universidad de Florida. 


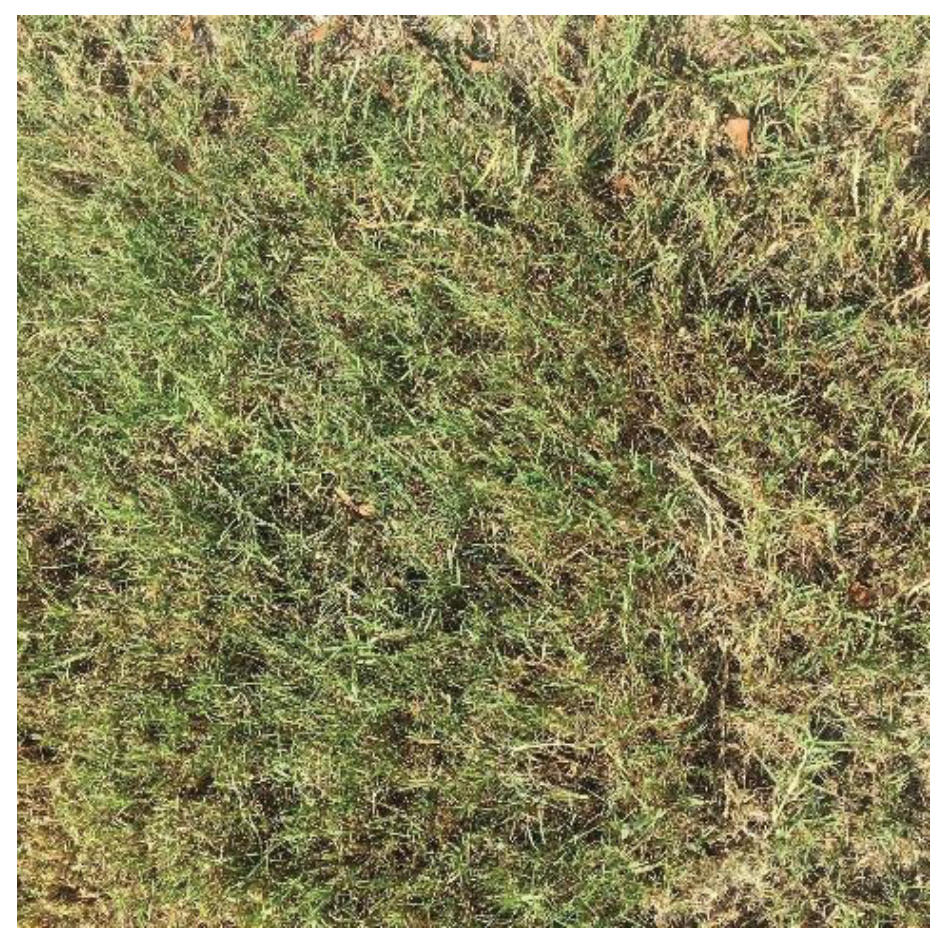

Figura 2. Grama Bermuda común.

Credits: L. Trenholm, UF/IFAS

\section{Mantenimiento de grama Bermuda en patios}

\section{Establecimiento}

La grama Bermuda se puede propagar a través de piezas cuadradas o rectangulares (sod), ramitas (sprigs), pedacitos o cuadritos (plugs) o semillas. Si el suelo está adecuadamente preparado y si se utilizan las prácticas de propagación correctas, cualquiera de estos métodos resultará exitoso. Para informaciones detalladas sobre la propagación del pasto en el patio lean la publicación LH013, Establishing Your Florida Lawn (https://edis.ifas.ufl.edu/publication/ LH013).

La grama Bermuda se puede propagar en cualquier época del año en la mayor parte de Florida, aunque en el norte de Florida la mejor época para propagar esta especie es la primavera o los primeros meses del verano. El motivo es que hay que darle suficiente tiempo al pasto antes que empiecen los meses más fríos en los cuales hay menor crecimiento. Las semillas se pueden sembrar sin riesgos hasta el final del año a pesar de que el crecimiento esté reducido en el otoño. Cuando el pasto se esté propagando, es importante regar más frecuentemente y por un tiempo más corto que lo normal. Riegos múltiples y cortos por 7-10 días después de haber plantado el pasto ayudarán el establecimiento evitando la sequía. En los siguientes 7-10 días se debe regar una vez al día entregando $1 / 4-1 / 2$ pulgada de agua. Después de este período, la frecuencia del riego se puede reducir a 2-3 veces por semana, siempre entregando $1 / 4-1 / 2$ pulgada de agua. Tres o cuatro semanas después de haberse propagado, el pasto tendría que estar establecido, y se podrá regar según sus necesidades. Para más informaciones, lean ENH9 Watering Your Florida Lawn (https://edis. ifas.ufl.edu/publication/LH025).

Un patio recién propagado no tendría que estar fertilizado por un mínimo de 30-60 días. Después de 60 días, el pasto habrá establecido un sistema radical activo, capaz de absorber a los nutrientes con un riesgo mínimo para el impacto ambiental. Demorar la primera fertilización no afectará la salud del pasto ni su propagación. El pasto no se deberá cortar hasta que las raíces tengan la oportunidad de anclarse (arraigarse) al suelo, normalmente 14-21 días después de la propagación. Las raíces están establecidas en el suelo cuando el pasto no se puede levantar sin hacer un esfuerzo considerable.

\section{PROPAGACIÓN POR RAMITAS (SPRIGGING)}

El método más común y económico para establecer la grama Bermuda es la propagación por ramitas. Las ramitas se distribuyen mecánicamente en grandes áreas, o a mano en áreas pequeñas. Las ramitas consisten en rizomas y estolones que tienen por lo menos dos nudos o junturas. Las ramitas se diseminan a una tasa de 200 a 400 bushel por acre o de 5 a 10 bushel por 1000 pies cuadrados, y luego se deben apretar al suelo. Este método es más barato que la propagación por piezas cuadradas o rectangulares, pero no produce la cobertura instantánea como lo hacen las piezas.

\section{PROPAGACIÓN POR PIEZAS CUADRADAS O RECTANGULARES (SODDING)}

Lapropagación por piezas cuadradas o rectangulares produce una superficie instantánea cubierta por pasto. Las piezas tienen que estar colocadas encima de suelo desnudo que ha sido mojado previamente; las piezas se tendrán que poner escalonadas, como su fueran ladrillos, con los bordes bien pegados para evitar huecos. El pasto se tiene que pisar y regar para asegurar buen contacto con el suelo y favorecer el crecimiento radical. Las piezas se tienen que regar dos o más veces por día con 1/4 de pulgada de agua, hasta que las raíces se hayan enganchado al suelo (normalmente 2 o 3 semanas). Después que se haya formado un buen aparato radical, la frecuencia del riego se reducirá y el tiempo de riego aumentará o será cambiado según las necesidades del pasto. 


\section{PROPAGACIÓN POR PEDACITOS O CUADRADITOS (PLUGGING)}

Las piezas se pueden cortar en cuadraditos con una cortadora utilizada en los greens de campos de golf (cup cutter) o con un machete. El espacio entre los cuadraditos varia entre 12 y 24 pulgadas, con el espacio inferior que llegará a cobertura total entre los 3 y 6 meses, y el superior entre 6 y 9 meses.

\section{SIEMBRA}

Solo la Bermuda común se puede sembrar. Las semillas se tienen que sembrar a una tasa entre 1 o 2 libras de semilla descascarada cada 1000 pies cuadrados.

\section{Fertilización}

Un programa de fertilización apropiado es muy importante para mantener el césped sano. La fertilización, junto a otras prácticas culturales, influencia la salud y la calidad del césped y reduce su vulnerabilidad a varios estreses incluyendo malezas, insectos y enfermedades. Es muy importante que quien fertilice el césped se familiarice y siga las mejores prácticas de manejo del programa FloridaFriendly Landscaping ${ }^{\text {rx }}$ Best Management Practices (FFL, $\mathrm{BMP}$ ). Estas prácticas se diseñaron para mantener el césped saludable, así como reducir cualquier contaminación de los recursos hídricos ocasionada por el mal uso de fertilizantes en patios. En el estado de Florida y en algunos condados y ciudades existen regulaciones locales que afectan la utilización de fertilizantes en el césped. Asegúrese de conocer estas regulaciones y siempre seguir las instrucciones de la etiqueta del fertilizante. Para más información sobre el Manejo Integrado de Plagas por favor refiérase a la publicación ENH979, Homeowner Best Management Practices for the Home Lawn (https://edis.ifas.ufl.edu/publication/ EP236).

A su vez, se puede utilizar un análisis de suelo para determinar si es necesario algún ajuste, y si este debe ocurrir antes o después de la propagación. La mayoría de los laboratorios que testean suelos pueden proveer recomendaciones sobre el pH y la salinidad del suelo. Si se siguen estas recomendaciones se podrán observar respuestas positivas en el césped. Testear el suelo también provee al usuario con recomendaciones para fósforo, pero, dado que los suelos de Florida normalmente tienen alto contenido de fósforo, los resultados del testeo a menudo indican que su aplicación no es necesaria. Hasta el momento no hay evidencia que justifique el testeo de suelo para decidir si aplicar otros nutrientes al césped.
La Universidad de Florida recomienda aplicar $1 / 2$ libra o 1 libra de nitrógeno cada 1000 pies cuadrados de césped por aplicación de fertilizante, y no más de 0.7 libras de nitrógeno soluble en cada aplicación. La regulación de Florida (Florida Rule, 5E-1.003) permite la aplicación de hasta 2 libras de nitrógeno cada 1,000 pies cuadrados cuando se aplica el fertilizante en primavera o verano, y si se usa un fertilizante con por lo menos un 65\% de fertilizante de nitrógeno de liberación controlada. Para información de cómo determinar la cantidad de fertilizante requerida, por favor refiérase a la publicación ENH962, Figuring Out Fertilizer for the Home Lawn (https://edis.ifas. ufl.edu/publication/EP221).

Como regla general, la primera aplicación de fertilizante en el año debe de ser a principios de abril en el centro de Florida y a mediados de abril en el norte de Florida. Aplicaciones de nitrógeno hechas justo antes de la última helada podrían resultar en césped dañado. En el sur de Florida, pueden hacerse en cualquier momento del año ya que el crecimiento ocurre ininterrumpidamente durante todo el año. Las guías para fertilización de la Universidad de Florida ofrecen una serie de cantidades y proporciones de nutrientes por las cuales una especie de césped en particular puede ser exitosamente mantenida en las diferentes regiones del estado. Estas cantidades incluyen el efecto que determinados microclimas locales puedan tener en el crecimiento del césped. Un ejemplo sería un césped que se encuentra parcialmente a la sombra y al sol. El césped que crece en la sombra necesita menos fertilizante que uno creciendo al sol. La fertilización también está afectada por el tipo de suelo, la cantidad de materia orgánica y el manejo de los residuos de césped cortado (clippings). Reciclar los residuos de césped cortado restituye algunos nutrientes al césped y podría reducir las necesidades de fertilización. Adicionalmente, un césped nuevo en un suelo arenoso con poca materia orgánica requiere más abono que uno que ha sido abonado por años. En Florida, algunas casas y zonas nuevas pueden estar contiguas a zonas mucho más viejas, por lo que la misma cantidad de abono pueda que no sea buena para todas.

Las guías de fertilización dividen al estado en tres áreas geográficas como se ve en la Tabla 1. Las cantidades están expresadas en libras de nitrógeno cada 1000 pies cuadrados por año. Para información de cómo determinar la cantidad de fertilizante requerida, por favor refiérase a la publicación ENH962, Figuring Out Fertilizer for the Home Lawn (https://edis.ifas.ufl.edu/publication/EP221). 
Cuando el pH del suelo es superior a 7, o donde se riega con agua con $\mathrm{pH}$ elevado, la aparición de hojas amarillas pueden ser una indicación de falta de hierro o manganeso. Aplicaciones de materiales solubles o quelados pueden proveer un reverdecimiento en algunos casos. El hierro solo se tendría que aplicar a las láminas foliares, o como granular quelado.

\section{Corte}

Es necesario contar con prácticas apropiadas de corte para tener un césped saludable y atractivo. La altura y la frecuencia del corte tienen que ser ajustados por el nivel de mantenimiento del césped, y por la temporada. Con niveles de mantenimiento bajos o moderados, la grama Bermuda se debe cortar a una altura de 1 o 2 pulgadas, que implica uno o dos cortes por semana. Este manejo ayuda el césped a desarrollar un sistema radical profundo y da un aspecto más agradable. Con estos niveles de mantenimiento, la frecuencia del corte tendrá que ajustarse a la tasa de crecimiento del césped. Con niveles de manejo altos, la grama Bermuda se puede cortar a una altura de $1 / 2$ pulgada, pero esta altura necesitará cortes más frecuentes durante la época de crecimiento. Cortar a esta altura y frecuencia requiere aportes de fertilizantes y agua más grandes para mantener un aspecto atractivo y duradero. Cabe aclarar que alturas de corte bajas y niveles altos de mantenimiento predisponen el césped a tener problemas de malezas y enfermedades. A pesar del nivel de mantenimiento, no más de 1/3 de las hojas deben ser removidas en cualquier corte.

Una cortadora de carrete (reel mower) es preferible para cortar Bermuda de textura fina cortada a alturas inferiores a 1 pulgada. Estas cortadoras generan un corte más limpio y pueden ajustarse a alturas bajas. Los residuos de césped cortado se pueden dejar en el césped mantenido con bajos niveles de fertilización si el césped viene cortado a alturas y frecuencias apropiadas. Los residuos no contribuyen al acolchonamiento y aportan nutrientes adicionales. Los residuos del corte deben removerse solo si la cantidad de tales residuos es tan excesiva como para resultar en aglomerados, o si el aspecto del césped es más importante.

\section{Riego}

Un patio de Bermuda se debe regar "cuando se necesita" para que mantenga color verde. Sin embargo, la grama Bermuda puede persistir durante periodos de sequía prolongados, pero cambiará su color a marrón si entrará en hibernación. El césped se volverá marrón y parará de crecer durante el periodo de hibernación, pero se revivirá y empezará a crecer de nuevo ni bien se regará con suficientes aportes hídricos. El riego se necesita cuando las hojas comienzan a doblarse, ponerse de un color azul-grisáceo, o cuando la marca de los pies se ven al caminar sobre el césped. Aplique 1/2-3/4 pulgadas de agua por aplicación. Esto moja aproximadamente las 4-6 pulgadas superiores del suelo donde están la mayor cantidad de las raíces. Con el objetivo de determinar el tiempo de riego para suministrar la cantidad de agua adecuada, coloque varios recipientes cilíndricos con lados rectos (por ej.: latas de atún o de comida de gatos) a través de la zona a regar, riegue cada zona y mida cuánto tiempo pasa hasta llenar los recipientes a un nivel de 1/2-3/4 pulgadas, apunte el tiempo transcurrido. Cada zona probablemente usará tiempos distintos para aplicar la misma cantidad de agua. Programe los tiempos para cada zona utilizando el reloj del sistema de riego automático. Si la variación en los recipientes es alta, necesita examinar mejor el sistema para mejorar la uniformidad. Refiéranse a la publicación ENH61, How to Calibrate Your Sprinkler System (https://edis.ifas.ufl.edu/ publication/lh026).

\section{Manejo de plagas}

Las plagas más comunes incluyen a los insectos y los hongos que pueden dañar la grama Bermuda. Pueden encontrarse servicios de diagnóstico y recomendaciones sobre cómo lidiar con estas plagas en las oficinas de extensión de UF/IFAS.

\section{Nemátodos}

Las plagas más importantes para la grama Bermuda en Florida son los nemátodos. Los nemátodos pueden causar falta de color y debilitamiento del césped, especialmente durante periodos cálidos y secos. Estas plagas dañan mucho el césped, particularmente cuando está cultivado en suelos arenosos y con altos niveles de mantenimiento. A pesar de que algunos cultivares puedes tolerar los nemátodos mejor que otros, ningún cultivar es resistente a la infestación de los mismos. El control químico de nemátodos es muy limitado y normalmente se requiere de un aplicador comercial para estos productos. Para más información sobre nemátodos, referirse a ENY006, Nematode Management in Residential Lawns (https://edis.ifas.ufl.edu/publication/ ng039).

\section{Insectos}

Los grillos-topo (Scapteriscus spp.) son los insectos que más dañan la grama Bermuda. Otros insectos que pueden dañar la grama Bermuda son el gusano telarañoso (Herpetogramma phaeopteralis), el gusano soldado (Spodoptera spp.), el gusano medidor (Mocis spp.) y los ácaros del césped. Hay muchas opciones de productos químicos para 
controlar estas especies, pero estas deben ser usadas solo si es necesario, y siempre combinadas con prácticas culturales y de fertilización adecuadas.

\section{Enfermedades}

La grama Bermuda puede tener varias enfermedades incluyendo la mancha de dollar (Sclerotinia homoeocarpa), la mancha grande (Rhizoctonia solani) y Helminthosporium. Un programa cultural adecuado puede ayudar a minimizar la mayoría de las enfermedades, utilizando fungicidas solo parar curar los síntomas más graves. Se vea SS-PLP-14, Turfgrass Disease Management (https://edis.ifas.ufl.edu/ publication/LH040), para más información.

\section{Malezas}

Las malezas presentes en el césped de grama Bermuda son un síntoma de que el césped está debilitado por prácticas culturales inadecuadas, o por enfermedades. Para más información refiérase a la publicación ENH884, Weed Management Guide for Florida Lawns (https://edis.ifas.ufl.edu/ publication/EP141). Prácticas de mantenimiento adecuadas pueden reducir la mayor parte de los problemas de malezas. Si las malezas se convierten en un problema persistente, se podrán utilizar herbicidas específicos registrados para la grama Bermuda en pre-emergencia o post-emergencia.

Tabla 1. Recomendaciones de fertilización anual para grama Bermuda en las tres regiones de Florida.

\begin{tabular}{|c|c|}
\hline Localización ${ }^{1}$ & $\begin{array}{c}\text { Guía de fertilización con } \mathbf{N} \\
\text { (Libras de N/1000 pies cuadrados por año) }\end{array}$ \\
\hline Norte de Florida & $3-5$ \\
\hline Florida Central & $4-6$ \\
\hline Sur de Florida & $5-7$ \\
\hline
\end{tabular}

${ }^{1}$ Florida norte-De Ocala hacia el norte; Centro-Del sur de Ocala hasta una línea que va de Vero Beach a Tampa; Sur-todo lo que queda al sur de esta línea.

${ }^{2}$ Estas tasas son probablemente más altas que las que realmente se necesitan para patios. Se requiere de más investigación para ajustarlas. 